\title{
Histopathological examination of tissue resected during bariatric procedures - to be done or not to be done?
}

\author{
Maciej Walędziak ${ }^{1}$, Anna Różańska-Walędziak ${ }^{2}$, Piotr K. Kowalewskii ${ }^{1}$, Michał R. Janik ${ }^{1}$, Krzysztof Paśnik $^{1}$ \\ ${ }^{1}$ Department of General, Oncological, Metabolic and Thoracic Surgery, Military Institute of Medicine, Warsaw, Poland \\ ${ }^{2} 2^{\text {nd }}$ Department of Obstetrics and Gynecology, Medical University of Warsaw, Warsaw, Poland
}

Videosurgery Miniinv 2017; 12 (2): 135-139

DOI: https://doi.org/10.5114/wiitm.2017.67807

\begin{abstract}
Introduction: Obesity is one of the major lifestyle diseases and provokes various comorbidities, such as hypertension, type 2 diabetes mellitus, obstructive sleep apnea, and even neoplasms. Bariatric surgery is the most effective treatment of obesity. Since cost-effectiveness has become a major concern, there is a tendency to avoid general histological evaluation of surgical specimens during routine procedures.

Aim: To evaluate the necessity of histopathological investigation of tissue excised during bariatric surgery and to verify whether the operation should be continued in the case of suspicious macroscopic findings.

Material and methods: From January 2013 to December 2016, 1252 patients with obesity were qualified for bariatric procedures. The qualification was performed according to the current European recommendations. Every operation started with an inspection of the peritoneal cavity performed once the abdomen was insufflated. If a macroscopic pathology was found, the specimen was secured for histopathological investigation.

Results: Out of $81(6.47 \%)$ patients from whom histopathological samples were collected, $39 \%(n=32)$ showed negative results, and $61 \%(n=49)$ cases showed abnormalities.

Conclusions: As it is impossible to exclude the existence of gastric tumors only in preoperative gastroscopy and ultrasonography, especially as there is a higher risk in obese patients, routine histological examination of tissue excised during bariatric procedures should be considered. Since most of the neoplasms were found to be benign, there is no need to abandon the bariatric procedure if a pathology is found and resected. Bariatric surgeons should always focus on thorough examination of the abdominal and the pelvic cavity, especially in female patients.
\end{abstract}

Key words: obesity, bariatric surgery, incidental findings.

\section{Introduction}

Obesity is one of the major lifestyle diseases and provokes various comorbidities, such as hypertension (HTN) [1], type 2 diabetes mellitus (T2DM) [2], obstructive sleep apnea (OSA), metabolic syndrome, and even neoplasms [3, 4]. Bariatric surgery is the most effective treatment of obesity, surpassing non-surgical methods, such as diet, exercise or pharmacology [5-7]. Every patient suffering from obesity with body mass index (BMI) over $40 \mathrm{~kg} / \mathrm{m}^{2}$ or with BMI over $35 \mathrm{~kg} / \mathrm{m}^{2}$ with comorbidities may be qualified for bariatric surgery. American and European bariatric federations are introducing the idea of lowering the qualifying level of BMI [8]. In 2013 over 450000 bariatric procedures were performed worldwide, and these numbers are constantly increasing [9]. In Poland, as stated by Janik et al. [10], we can observe a similar trend, with over 1500 procedures performed in 2014. Based on this fact, many bariat-

\section{Address for correspondence}

Maciej Walędziak MD, Department of General, Oncological, Metabolic and Thoracic Surgery, Military Institute of Medicine,

128 Szaserów St, 04-141 Warsaw, Poland, phone: +48 606387 636, e-mail: maciej.waledziak@gmail.com 
ric centers are introducing the Enhanced Recovery After Surgery (ERAS) protocol to shorten the length of stay (LOS) and improve the quality of treatment [11]. Many surgical departments are waiving gastroscopy and abdominal ultrasonography (US) as standard qualifying procedures $[12,13]$. Since cost-effectiveness has become a major concern, there is a tendency to avoid general histological evaluation of surgical specimens during routine procedures, such as appendectomy, cholecystectomy or hemorrhoidectomy [14-16]. The same concerns apply to bariatric surgery. In recent years, several studies have acknowledged incidental histological findings after bariatric procedures, yet they mostly focused on single types of surgical procedures $[17,18]$ or had a small number of presented cases $[19,20]$.

\section{Aim}

The aim of this study was to evaluate the necessity of histopathological investigation of tissue excised during bariatric surgery and to verify whether the operation should be continued in the case of suspicious macroscopic findings.

\section{Material and methods}

From January 2013 to December 2016, 1252 patients with obesity were qualified for bariatric procedures. The qualification was performed according to the current European and American recommendations: BMI greater than or equal to $40 \mathrm{~kg} / \mathrm{m}^{2}$, or BMI greater than or equal to $35 \mathrm{~kg} / \mathrm{m}^{2}$ with at least one serious obesity-related comorbidity, such as heart disease, T2DM or severe OSA [8]. Every patient had the basic blood parameters measured, as well as endoscopy of the upper gastrointestinal tract and ultrasound examination of the abdominal cavity. If any of the results were abnormal, additional tests were performed. If the patient suffered from active Helicobacter pylori infection, eradication and subsequent endoscopy after treatment were performed. Patients were qualified by the consultants for laparoscopic sleeve gastrectomy (LSG), duodenal switch (DS) or Roux-en-Y gastric bypass (LRYGB) according to their BMI, alimentary habits and co-morbidities. The operative protocols were standardized and performed by bariatric surgeons.

Every operation started with an inspection of the peritoneal cavity, performed once the abdomen was insufflated. If there was any abnormal finding, a consultant made the decision whether to continue or abandon the procedure.

If a macroscopic pathology was found, the specimen was secured for histopathological investigation.

We collected data from the medical records regarding the patients' baseline characteristics and histopathological findings. Further analysis was performed in cases of discovered abnormalities.

The analysis included age, sex, BMI, type of procedure, origin of the sample, LOS and the histopathological result.

\section{Results}

From January 2013 to December 2016, 1252 morbidly obese patients were qualified for bariatric procedures.

Out of $81(6.47 \%)$ patients from whom histopathological samples were collected, $39 \%(n=32)$ showed negative results. In $61 \%(n=49)$ of cases the results were positive and showed abnormalities.

Out of 49 patients with positive results of histopathological analysis, 28 (57.14\%) were male. The mean age was $51.37 \pm 10.17$, from 31 to 69 years old. The mean BMI was $47.4 \pm 7.94 \mathrm{~kg} / \mathrm{m}^{2}$, from 33.39 to $72.76 \mathrm{~kg} / \mathrm{m}^{2}$. The mean length of stay in hospital was $4.43 \pm 1.04$, from 3 to 8 days.

In 31 cases LSG was performed, in 11 cases LRYGB, and in 7 cases the operation was ended after diagnostic laparoscopy and surgical biopsy, without performing the bariatric procedure.

Out of 49 samples, 29 came from the stomach: $16(1.28 \%)$ cases were found to be gastrointestinal stromal tumors (GISTs), 5 (0.40\%) leiomyomas, 3 (0.24\%) lipomas, 2 (0.16\%) fibromas, 2 (0.16\%) Schwannomas and 1 (0.08\%) neurofibroma. Sixteen specimens came from the liver: $4(0.32 \%)$ cholangiocellular adenomas (CCA), 4 (0.32\%) bile duct adenomas (BDA), $4(0.32 \%)$ cases of non-alcoholic steatohepatitis (NASH), 3 (0.24\%) cases of cirrhosis, and $1(0.08 \%)$ focal nodular hyperplasia (FNH). Three contained perigastric tissue: $1(0.08 \%)$ tuberculosis, $1(0.08 \%)$ sarcoidosis and $1(0.08 \%)$ simple cyst. One $(0.08 \%)$ came from the small intestine and it was a lipoma.

The results of pathology varied, as shown in Table I.

93.5\% of our patients who underwent bariatric surgery did not present any macroscopic clinical pathologies. The remaining $6.47 \%$ did have possible 
malignancies which required a surgical biopsy for further investigation. Out of these, 39.5\% did not reveal any microscopic pathologies. We found no malignant neoplasms.

The incidental macroscopic findings which led to abandoning the bariatric procedure originated from the liver (1 case of non-alcoholic steatosis cirrhosis and 1 bile duct adenoma), stomach (1 fibroma and 1 leiomyoma) and perigastric tissue (1 sarcoidosis and 1 simple cyst).

\section{Discussion}

Bariatric surgery has become the most popular and efficient method of treatment for obesity and its comorbidities. In many cases surgery is the patient's only chance for recovery and improved quality of life.

Ohanessian et al. [17] examined every gastric specimen from 310 sleeve gastrectomies. Two hundred and fourteen (69\%) cases presented no pathology the remaining minority had histologic findings ranging from the more common chronic gastritides to less common but very significant GIST. Benign stromal tumors (GISTs) were found in $3(1 \%)$ cases. Only 8 patients from the study underwent a pre-op gastroscopy. Therefore, based on the higher GIST rate, the authors suggest a routine microscopic evaluation of the gastric specimen. Nine liver biopsies were done without finding any pathologies other than cirrhosis or steatosis.

Almazeedi et al. [18] investigated 656 specimens from sleeve gastrectomies. They found 488 (74.4\%) cases of chronic gastritis and $5(0.2 \%)$ cases of malignant tumors - despite thorough endoscopic evaluation and $H$. pylori eradication before the surgery. The authors recommend a routine endoscopic evaluation prior to LSG.

Greenbaum and Friedel [19] focused on the thorough examination of the peritoneum, pelvic cavity and genitals before commencing bariatric surgery. While analyzing 400 cases they found 25 ovarian pathologies and 3 malignant tumors. Histopathological results from the specimen retrieved for these procedures influenced further medical treatment.

Analyzing 546 cases of specimens from sleeve gastrectomies, AbdullGaffar et al. [21] found only one benign neoplasm, but no malignant pathologies. The authors suggest waiving pre-op gastroscopy and further histological investigation only when the gas-
Table I. Pathology results of resected specimens

\begin{tabular}{|ll|}
\hline Stomach & $16(1.28 \%)$ gastrointestinal stromal tumors (GISTs) \\
& $5(0.40 \%)$ leiomyomas \\
& $3(0.24 \%)$ lipomas \\
& $2(0.16 \%)$ fibromas \\
& $2(0.16 \%)$ Schwannomas \\
& $1(0.08 \%)$ neurofibroma \\
\hline Liver & $4(0.32 \%)$ cholangiocellular adenomas (CCA) \\
& $4(0.32 \%)$ bile duct adenomas (BDA) \\
& $4(0.32 \%)$ non-alcoholic steatohepatitis (NASH) \\
& $3(0.24 \%)$ cirrhosis \\
& $1(0.08 \%)$ focal nodular hyperplasia (FNH) \\
\hline Perigastric & $1(0.08 \%)$ tuberculosis \\
tissues & $1(0.08 \%)$ sarcoidosis \\
& $1(0.08 \%)$ simple cyst \\
\hline Small & $1(0.08 \%)$ lipoma \\
intestine & \\
\hline
\end{tabular}

tric specimen arouses suspicion of pathology. They recommend adjusting the surgical approach to the local epidemiology of gastric cancer.

Among 427 gastric specimens from open gastric bypass Sohn et al. [22] found only chronic gastritis, 1 leiomyoma and 1 cyst. The authors suggested performing a microscopic evaluation depending on the intraoperative view rather than making it a routine procedure.

Out of 145 cases of sleeve gastrectomy examined by Clapp [23], none revealed any pathologies, apart from several cases of chronic gastritis, 1 leiomyoma and 3 hyperplastic polyps.

Finnell et al. [20] examined 398 specimens from bariatric procedures, reporting 9 cases of incidental findings: 4 benign tumors (2 GISTs and 2 leiomyomas), 2 cases of ectopic pancreatic tissue, 1 bile duct adenoma (BDA), 1 vascular malformation and 1 hemangioma. The authors recommend a thorough investigation of the peritoneum and pelvic area, but at the same time encourage continuation of the procedure, even if any incidental pathologies are found. Most of the specimens turned out to be benign.

Benign liver neoplasms are rarely found, most often being revealed incidentally during autopsy in $0.01 \%$ to $0.6 \%$ of cases - cholangiocellular adenomas (CAA) $0.3 \%$, BDA $0.3 \%$, focal nodular hyperplasia (FNH) $0.07 \%$ [24].

Ohanessian, Almazeedi and Greenbaum suggest routine histopathological examination of tissue excised during bariatric procedures as being of high 
diagnostic value in the case of presence of pathologies that had not been found before the operation. AbdullGaffar and Sohn present an opposite approach, recommending examining the abdominal cavity and excised gastric specimens as a routine procedure, and proceeding to histopathological examination only if there are pathological macroscopic findings. According to most authors, performing a laparoscopy, even as a bariatric procedure, should always lead to thorough examination of the whole abdominal and pelvic cavity to avoid omitting any pathologies.

As the opinions differ among the authors, the issue should be subject to further examination. Based on the specimens collected in our study and following the opinions of the majority of authors, our suggestion is to recommend routine examination of gastric tissue excised during bariatric procedures and biopsy in the case of pathologies found by exploration of the abdominal cavity, as well as continuing the procedure even when pathologies are revealed, as they tend to be found benign.

\section{Conclusions}

As it is impossible to exclude the existence of gastric tumors only in preoperative gastroscopy and ultrasonography, especially as there is a higher risk in obese patients, routine histological examination of tissue excised during bariatric procedures should be considered.

Since most of the neoplasms were found to be benign, there is no need to abandon the bariatric procedure if a pathology is found and resected.

Every laparoscopic bariatric procedure should simultaneously be a diagnostic laparoscopy, focused on thorough examination of the abdominal and the pelvic cavity, especially in female patients.

\section{Conflict of interest}

The authors declare no conflict of interest.

\section{References}

1. Landsberg L, Aronne LJ, Beilin LJ, et al. Obesity-related hypertension: pathogenesis, cardiovascular risk, and treatment: a position paper of The Obesity Society and the American Society of Hypertension. J Clin Hypertens (Greenwich) 2013; 15: 14-33.

2. Hartemink N, Boshuizen HC, Nagelkerke NJ, et al. Combining risk estimates from observational studies with different exposure cutpoints: a meta-analysis on body mass index and diabetes type 2. Am J Epidemiol 2006; 163: 1042-52.
3. Kaaks R, Lukanova A, Kurzer MS. Obesity, endogenous hormones, and endometrial risk: a synthetic review. Cancer Epidemiol Biomarkers Prev 2002; 11: 1531-43.

4. Guh DP, Zhang W, Bansback N, et al. The incidence of co-morbidities related to obesity and overweight: a systematic review and meta-analysis. BMC Public Health 2009; 9: 88.

5. Colquitt JL, Pickett K, Loveman E, et al. Surgery for weight loss in adults. Cochrane Database Syst Rev 2014; 8: CD003641.

6. Lindekilde N, Gladstone BP, Lubeck M, et al. The impact of bariatric surgery on quality of life: a systematic review and meta-analysis. Obes Rev 2015; 16: 639-51.

7. Gloy VL, Briel M, Bhatt DL, et al. Bariatric surgery versus non-surgical treatment for obesity: a systematic review and meta-analysis of randomised controlled trials. BMJ 2013; 347: f5934.

8. Neylan CJ, Kannan U, Dempsey DT, et al. The surgical management of obesity. Gastroenterol Clin North Am 2016; 45: 689703.

9. Angrisani L, Santonicola A, Iovino P, et al. Bariatric surgery worldwide 2013. Obes Surg 2015; 25: 1822-32.

10. Janik MR, Stanowski E, Paśnik K. Present status of bariatric surgery in Poland. Videosurgery Miniinv 2016; 11: 22-5.

11. Małczak P, Pisarska M, Piotr M, et al. Enhanced recovery after bariatric surgery: systematic review and meta-analysis. Obes Surg 2017; 27: 226-35.

12. De Palma GD, Forestieri P. Role of endoscopy in the bariatric surgery of patients. World J Gastroenterol 2014; 20: 7777-84.

13. Petrick A, Benotti P, Wood GC, et al. Utility of ultrasound, transaminases, and visual inspection to assess nonalcoholic fatty liver disease in bariatric surgery patients. Obes Surg 2015; 25 : 2368-75.

14. Matthyssens LE, Ziol M, Barrat C, Champault GG. Routine surgical pathology in general Surgery. Br J Surg 2006; 93: 362-8.

15. Swank HA, Eshuis EJ, Ubbink DT, Bemelman WA. Is routine histopathological examination of appendectomy specimens useful? A systematic review of the literature. Colorectal Dis 2011; 13: $1214-21$

16. Lohsiriwat V, Vongjirad A, Lohsiriwat D. Value of routine histopathologic examination of three common surgical specimens: appendix, gallbladder, and hemorrhoid. World J Surg 2009; 33: 2189-93.

17. Ohanessian SE, Rogers AM, Karamchandani DM. Spectrum of gastric histopathologies in severely obese American patients undergoing sleeve gastrectomy. Obes Surg 2016; 26: 595-602.

18. Almazeedi S, Al-Sabah S, Al-Mulla A, et al. Gastric histopathologies in patients undergoing laparoscopic sleeve gastrectomies. Obes Surg 2013; 23: 314-9.

19. Greenbaum D, Friedel D. Unanticipated findings at bariatric surgery. Surg Obes Relat Dis 2005; 1: 22-4.

20. Finnell CW, Madan AK, Ternovits CA, et al. Unexpected pathology during laparoscopic bariatric surgery. Surg Endosc 2007; 21: 867-9.

21. AbdullGaffar B, Raman L, Khamas A, AlBadri F. Should we abandon routine microscopic examination in bariatric sleeve gastrectomy specimens? Obes Surg 2016; 26: 105-10.

22. Sohn VY, Arthurs ZM, Martin MJ, et al. Incidental pathologic findings in open resectional gastric bypass specimens with 
routine cholecystectomy and appendectomy. Surg Obes Relat Dis 2008; 4: 608-11.

23. Clapp B. Histopathologic findings in the resected specimen of a sleeve gastrectomy. JSLS 2015; 19: e2013.00259.

24. Ignee A, Piscaglia F, Ott M, et al. A benign tumour of the liver mimicking malignant liver disease - cholangiocellular adenoma. Scand J Gastroenterol 2009; 44: 633-6.

Received: 31.01.2017, accepted: 12.04.2017. 